\title{
Esôfago de Barrett e displasia: critérios diagnósticos
}

\author{
Barret's esophagus and dysplasia: diagnostic criteria
}

\begin{tabular}{l|l}
\multicolumn{1}{c|}{ Unitermos } & resumo \\
Esôfago de Barrett & $\begin{array}{l}\text { A presente revisão focaliza aspectos conceituais e os principais problemas diagnósticos referentes ao } \\
\text { esôfago de Barrett e à displasia. O esôfago de Barrett resulta de complicação da doença do refluxo } \\
\text { gisplasia }\end{array}$ \\
$\begin{array}{l}\text { Diagnóstico } \\
\text { no esôfago tubular acima da junção esofagogástrica. Histologicamente, é caracterizado pela substituição } \\
\text { do epitélio estratificado pavimentoso por epitélio colunar especializado com células caliciformes, expres- } \\
\text { so como metaplasia intestinal. A importância biológica do esôfago de Barrett é o risco de progressão } \\
\text { para câncer. A displasia é o principal marcador biológico preditivo de evolução para adenocarcinoma. } \\
\text { Identificar e graduar a displasia constitui importante questão na prática diagnóstica. O diagnóstico } \\
\text { patológico do esôfago de Barrett deve conter informações sobre a investigação de displasia. O principal } \\
\text { diagnóstico diferencial da displasia é feito em relação a reatividade e regeneração epitelial no contexto } \\
\text { de inflamação da mucosa. Como a variabilidade de interpretação é um dos principais problemas no } \\
\text { diagnóstico da displasia, os casos de esôfago de Barrett devem ser enviados à consulta para segunda } \\
\text { opinião diagnóstica. O exame anatomopatológico é fundamental para definir o diagnóstico de esôfago } \\
\text { de Barrett e para rastrear a displasia, que é o principal marcador de risco para câncer nesta entidade. }\end{array}$ \\
\hline
\end{tabular}

abstract

This review focuses on conceptual aspects of Barrett's esophagus and Barrett's related dysplasia and highlights current diagnostic problems on the interpretation of these lesions. Barrett's esophagus results from longstanding gastroesophageal reflux. Endoscopically it is recognized by the presence of glandular mucosa in the tubular esophagus above the esophagogastric junction at variable lenghts. Histologically it is characterized by the presence of specialized columnar epithelium with globet cells expressed as intestinal metaplasia. The importance of Barret's esophagus is the risk for developing adenocarcinoma. Dysplasia is the main biomarker for the progression to adenocarcinoma. The pathologic diagnosis of Barrett's esophagus should contain information on the presence and grade of dysplasia. Distinguishing betweeen dysplasia and regenerative changes in the face of active inflammation may be difficult. Correct interpretation and grading of dysplasia is an important goal for pathologists, since it is a major factor on follow-up and treatment of the patients.

\author{
Maria Aparecida Marchesan Rodrigues
}

(2)




\section{Introdução}

O esôfago de Barrett (EB) é uma condição patológica adquirida, caracterizada pela substituição do epitélio estratificado pavimentoso do esôfago por epitélio colunar especializado do tipo intestinal|(3, 10-12). Ocorre em pacientes com doença do refluxo gastroesofágico de longa duração(30). Representa uma resposta adaptativa da mucosa à agressão pelo ácido. Os fatores de risco para desenvolvimento do EB são os que favorecem o refluxo gastroesofágico, e incluem a hérnia de hiato, a incompetência do esfíncter inferior do esôfago e o refluxo do conteúdo duodenogástrico ${ }^{(11,30)}$. A importância biológica do EB é o risco para câncer. Cerca de 10\% dos pacientes portadores de EB desenvolvem adenocarcinoma no terço inferior do esôfago ${ }^{(11,12)}$. As estimativas do risco para câncer são imprecisas, visto que não se conhece o número total de pacientes com EB em relação aos que desenvolvem adenocarcinoma ${ }^{(11)}$. No Brasil, a maioria dos estudos sobre o EB focaliza procedimentos de conduta e tratamento cirúrgico. Os aspectos epidemiológicos e os atributos morfológicos dessa entidade são pouco estudados(2).

\section{Aspectos endoscópicos}

Endoscopicamente o EB é caracterizado pela presença de mucosa glandular no esôfago tubular acima da junção esofagogástrica ${ }^{(30)}$. É designado $E B$ curto quando o epitélio glandular é restrito aos $3 \mathrm{~cm}$ distais do esôfago tubular, e $E B$ clássico, ou de segmento longo, quando a mucosa glandular ocupa mais de $3 \mathrm{~cm}$ em direção cranial no esôfago tubular ${ }^{(20)}$. Portanto, no EB a junção escamocolunar encontra-se acima da junção esofagogástrica em extensões variáveis. A identificação endoscópica do $E B$ pode ser difícil, especialmente no EB curto, por não haver limites anatômicos que definam a região do esfíncter inferior do esôfago ${ }^{(30)}$. A presença de hérnia de hiato que acompanha com freqüência o EB dificulta a identificação da junção esofagogástrica e a caracterização do $\mathrm{EB}^{\left({ }^{(30)} \text {. Às }\right.}$ vezes, nem o endoscopista nem o patologista são capazes de identificar o local preciso da biópsia em relação ao esfíncter inferior do esôfago. Por isso, chegou-se ao consenso de que a presença de epitélio colunar especializado com metaplasia intestinal no esôfago tubular é o atributo morfológico que caracteriza o $E B^{(11,12)}$. Com esse critério diagnóstico a localização precisa da biópsia no exame endoscópico tornou-se irrelevante.

\section{Diagnóstico histológico}

Os estudos preliminares sobre o diagnóstico histológico do EB descreveram três tipos de epitélio como integrantes do espectro das alterações histopatológicas do $\mathrm{EB}^{(25}$ : o epitélio gástrico fúndico com células parietais e principais, o epitélio gástrico juncional da cárdia com glândulas secretoras de muco e o epitélio colunar especializado com metaplasia intestinal. Como somente a metaplasia intestinal correlacionou-se com a progressão para câncer ${ }^{(11)}$, o consenso atual para o diagnóstico histológico do EB é a presença de epitélio colunar especializado com células caliciformes, caracterizado morfologicamente como metaplasia intestinal (Figura 1).

O método histoquímico do Alcian blue $\mathrm{pH} 2,5$ cora em azul as mucinas ácidas (sialo e sulfomucinas) produzidas pelas células caliciformes, caracterizando histoquimicamente o fenótipo intestinal metaplásico ${ }^{(11,16)}$. As células colunares do revestimento foveolar produzem mucinas neutras que se coram em vermelho pelo ácido p-aminossalicílico (PAS). Ocasionalmente, células colunares foveolares coram-se em azul pelo Alcian blue. Essas células não são consideradas atributos morfológicos para o diagnóstico de EB. Representam fenótipo de transição entre 0 epitélio gástrico foveolar e o epitélio intestinal metaplásico ${ }^{(21)}$. Recomendase o uso do método do Alcian blue na rotina diagnóstica do $E B$, visto que aumenta a sensibilidade diagnóstica do epitélio intestinal metaplásico. Como o aspecto histológico do epitélio intestinal metaplásico do EB é similar ao da metaplasia intestinal encontrada na mucosa gástrica ou na cárdia, estudos utilizando métodos imunoistoquímicos investigaram a possibilidade de um fenótipo diferencial. Ormsby et al.(22) estudaram a expressão das citoceratinas 7 e 20 em peças cirúrgicas com EB longo e compararam

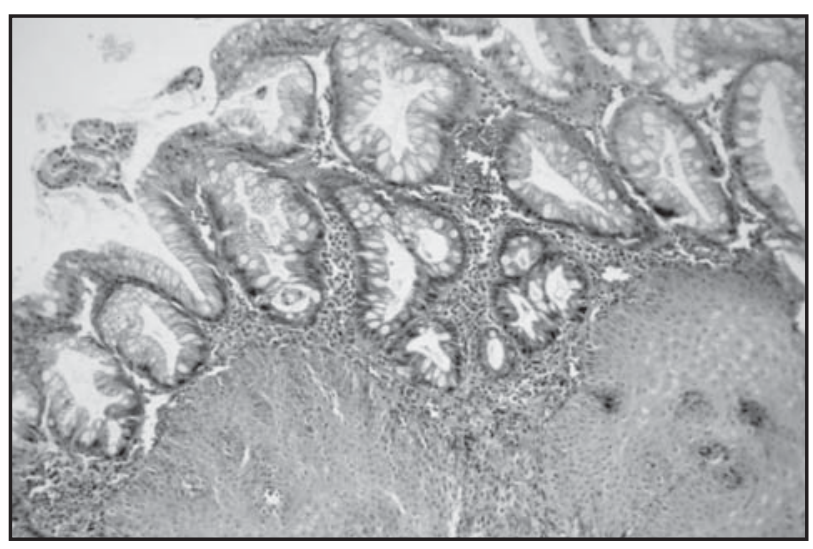

Figura 1 - Esôfago de Barrett negativo para displasia. A maturação epitelial está preservada. A presença de células caliciformes define o diagnóstico de esôfago de Barrett (HE 200x) 
com peças de estômago apresentando metaplasia intestinal na mucosa do antro. Todos os casos de EB longo apresentaram imunorreatividade superficial e profunda para CK7 e coloração superficial para CK20. Em contraste, os casos de metaplasia intestinal da mucosa gástrica não apresentaram imunorreatividade para CK7 e exibiram coloração superficial e profunda para CK20 nas áreas de metaplasia intestinal completa. A seguir, os pesquisadores investigaram ser a expressão diferencial das citoceratinas 7 e 20 útil para distinguir o epitélio intestinal metaplásico do EB da metaplasia intestinal da cárdia. Observaram que o fenótipo da metaplasia intestinal da cárdia era similar ao da mucosa gástrica e distinto do padrão CK7/20 expresso no epitélio intestinal metaplásico do $\mathrm{EB}^{(23)}$. Entretanto Glickman et $a l^{(7)}$ não encontraram diferenças entre casos de EB curto e metaplasia intestinal da cárdia quanto à expressão das citoceratinas 7 e 20. Estudos adicionais utilizando técnicas ancilares são necessários para investigar se é possível distinguir o fenótipo metaplásico do EB da metaplasia intestinal da cárdia, posto que fazem parte de processos patológicos distintos.

\section{Diagnóstico diferencial do esôfago de Barrett}

Os principais diagnósticos diferenciais do EB são a hérnia de hiato, a heterotopia de mucosa gástrica no esôfago e a metaplasia intestinal na junção esofagogástrica. Na hérnia de hiato, as biópsias demonstram mucosa gástrica de padrão corpo fundo ou da cárdia. A heterotopia de mucosa gástrica no esôfago ocorre em cerca de $10 \%$ dos pacientes submetidos à endoscopia digestiva altaa ${ }^{(4,13)}$. É mais freqüente no esôfago cervical e se expressa endoscopicamente como ilhas de mucosa glandular róseo-avermelhada em meio ao epitélio escamoso(4). O conhecimento do local preciso da biópsia é essencial para o diagnóstico correto dessa entidade, que resulta de um defeito da embriogênese na substituição do epitélio colunar pelo epitélio escamoso. Outro diagnóstico diferencial relevante e difícil é o da metaplasia intestinal da cárdia, que é histologicamente idêntica à metaplasia intestinal do $\mathrm{EB}^{(8)}$. A dificuldade é distinguir o EB curto da metaplasia intestinal da cárdia, pois o endoscopista nem sempre tem certeza se a biópsia foi retirada acima ou abaixo da junção esofagogástrica. A relevância é que os processos patológicos são distintos. A metaplasia intestinal da cárdia não é relacionada ao refluxo gastroesofágico e, portanto, aparentemente não se correlaciona com o risco para câncer no contexto da doença do refluxo gastroesofágico ${ }^{(8)}$. Há estudos que demonstram que a metaplasia intestinal da cárdia faz parte de quadro de pangastrite associada ao Helicobacter pylori(8). A análise imuno-histoquímica com a imunorreatividade superficial e profunda para CK20 e a ausência de expressão para CK7, que caracteriza o fenótipo de metaplasia intestinal completa na mucosa gástrica, podem ser úteis para identificar a metaplasia intestinal da cárdia, auxiliando no diagnóstico diferencial com o epitélio metaplásico do $E B^{(8)}$.

\section{Displasia no esôfago de Barrett}

Como o EB é condição pré-maligna, o papel do patologista no acompanhamento dos pacientes com EB é identificar a presença de displasia, que é o principal marcador biológico preditivo de evolução para adenocarcinoma ${ }^{(8,18,26)}$. A displasia é o atributo que orienta a conduta clínica nos pacientes com EB ${ }^{(28)}$, constituindo a expressão mais inicial do fenótipo neoplásico em nível morfológico ${ }^{(6,9,}$ 19). Estudos de carcinogênese demonstram que a displasia é a etapa que precede o aparecimento da neoplasia em nível macroscópico ${ }^{(14)}$. Corresponde, histologicamente, à neoplasia em localização intra-epitelial. Caracteriza-se por atipias celulares confinadas ao epitélio, sem invasão da membrana basal|(6,9,19,29). A graduação da displasia no EB é similar à proposta por Riedel|(29) para as doenças inflamatórias intestinais. Os graus utilizados são: displasia de baixo grau, displasia de alto grau e indefinido para displasia ${ }^{(9,17-19)}$. O diagnóstico de displasia é baseado em alterações citológicas e estruturais. $\mathrm{O}$ algoritmo proposto por Montgomery et al. ${ }^{(19)}$ permite distinguir alterações epiteliais indefinidas para displasia das alterações que correspondem à displasia de baixo grau ou de alto grau. $\mathrm{O}$ algoritmo baseia-se nas principais características histológicas encontradas no EB (Tabela). Deve ser aplicado a biópsias que apresentam os atributos morfológicos do $E B$, ou seja, o epitélio colunar com metaplasia intestinal. Os dois primeiros atributos do algoritmo são a maturação epitelial e a arquitetura das glândulas (Tabela), que devem ser analisadas no pequeno aumento do microscópio. A

\section{Tabela displasia no esôfago de Barrett \\ Algoritmo para graduação da}

1. Maturação epitelial (base das glândulas $x$ superfície)

2. Arquitetura da mucosa

3. Características citológicas

4. Inflamação, erosões, úlceras

Adaptado de MONTCOMERY, E. Pathology Case Reviews, v. 7, n. 1, p. 35-42, 2002. 
arquitetura da mucosa é a relação entre as glândulas e o estroma. A mucosa com arquitetura preservada exibe glândulas redondas ou ovais, sem ramificações, dispostas em meio a estroma abundante de tecido conjuntivo frouxo. Glândulas de aspecto normal aglomeradas são consideradas alteração estrutural discreta. $\mathrm{O}$ aglomeramento de glândulas de tamanhos e formas variadas com ramificações ou arranjos cribriformes constitui alteração arquitetural importante. A maturação epitelial é atributo-chave para o diagnóstico de displasia ${ }^{(11,19)}$. Deve ser analisada comparando-se as células das porções profundas da mucosa com as células superficiais. A mucosa com maturação preservada exibe, nas porções profundas, células imaturas com núcleos volumosos, hipercromáticos, por vezes pseudo-estratificados. Na superfície, as células apresentam núcleos menores, situados em posição basal e dispostos em monocamada. A relação núcleo/citoplasma exibe um gradiente da base para a superfície. A presença de núcleos proporcionalmente maiores na superfície é sinal de comprometimento da maturação epitelial. O terceiro atributo do algoritmo refere-se às características citológicas (Tabela), que devem ser analisadas nos aumentos maiores do

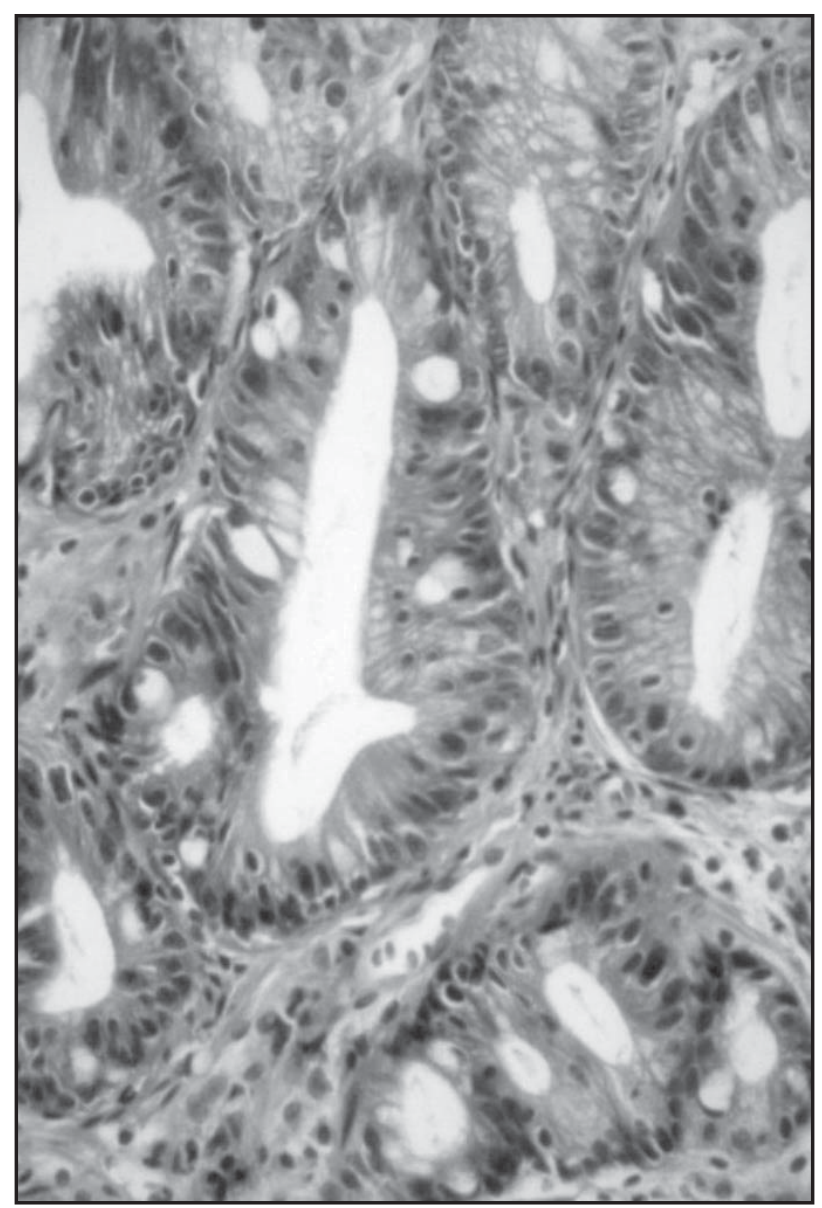

Figura 2 - Esôfago de Barrett com alterações epiteliais indefinidas para displasia. Núcleos com hipercromasia e estratificação focal (HE 400x) microscópio. Deve ser destacado que as células do EB geralmente apresentam atipias discretas, expressas por discreto aumento de volume dos núcleos, especialmente nas glândulas basais, visto que compreendem a forma incompleta da metaplasia intestinal. As atipias citológicas encontradas no EB podem corresponder à displasia ou à reatividade epitelial induzida pela inflamação. As células displásicas apresentam núcleos volumosos, hipercromáticos, com aumento da relação núcleo/citoplasma (Figuras 3 e 4). A polaridade dos núcleos que compreende a relação dos núcleos entre si pode estar mantida ou alterada. Considera-se polaridade normal quando os núcleos se dispõem em paralelo, com o maior eixo perpendicular à membrana basal (Figura $2 \mathrm{e}$ Figura 3). A perda de polaridade dos núcleos é atributo morfológico característico da displasia de alto grau. $\mathrm{O}$ quarto atributo do algoritmo refere-se à presença de inflamação, erosões e úlceras (Tabela), que induzem alterações citológicas e/ou estruturais exuberantes, sugestivas de displasia de baixo ou de alto grau, dificultando a interpretação diagnóstica. $\mathrm{O}$ diagnóstico anatomopatológico do esôfago de Barrett deve conter informações sobre a investigação de displasia.

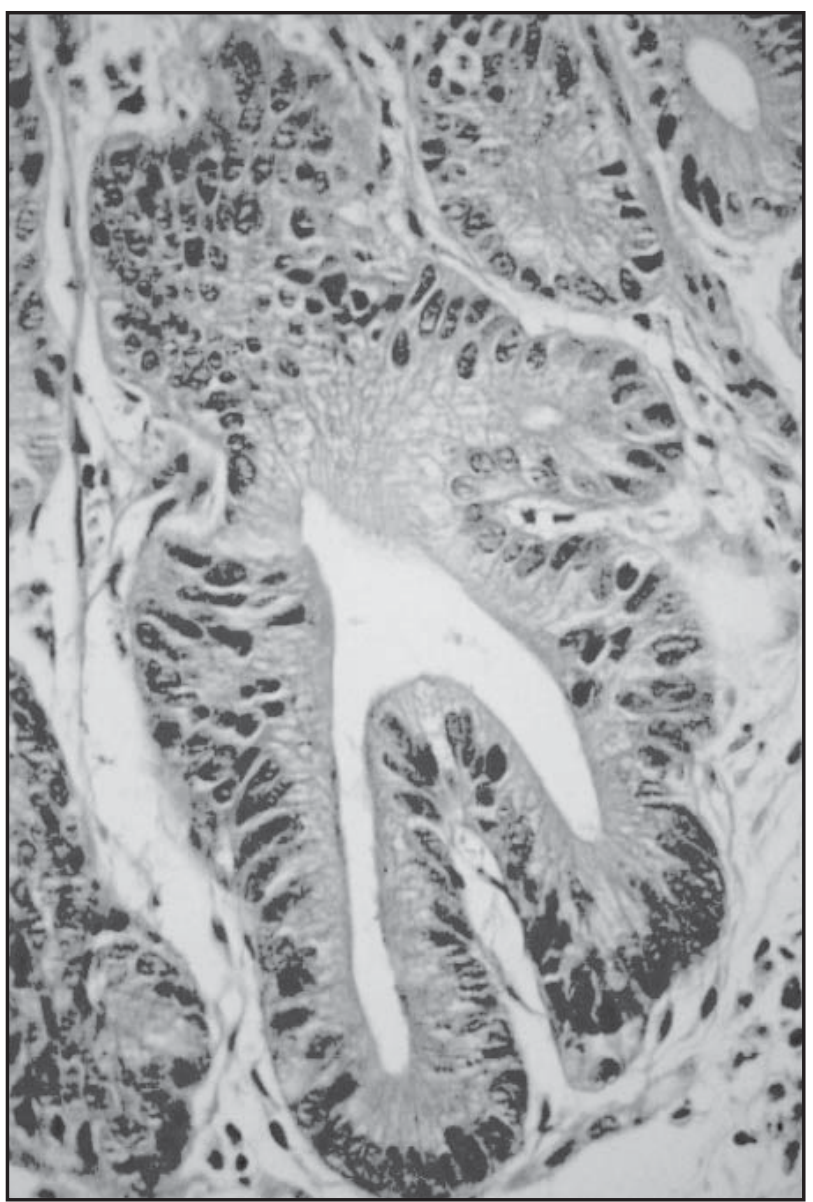

Figura 3 - Displasia de baixo grau. Núcleos com discariose e hipercromasia situados em posição basal. Há redução de células caliciformes (HE 400x) 


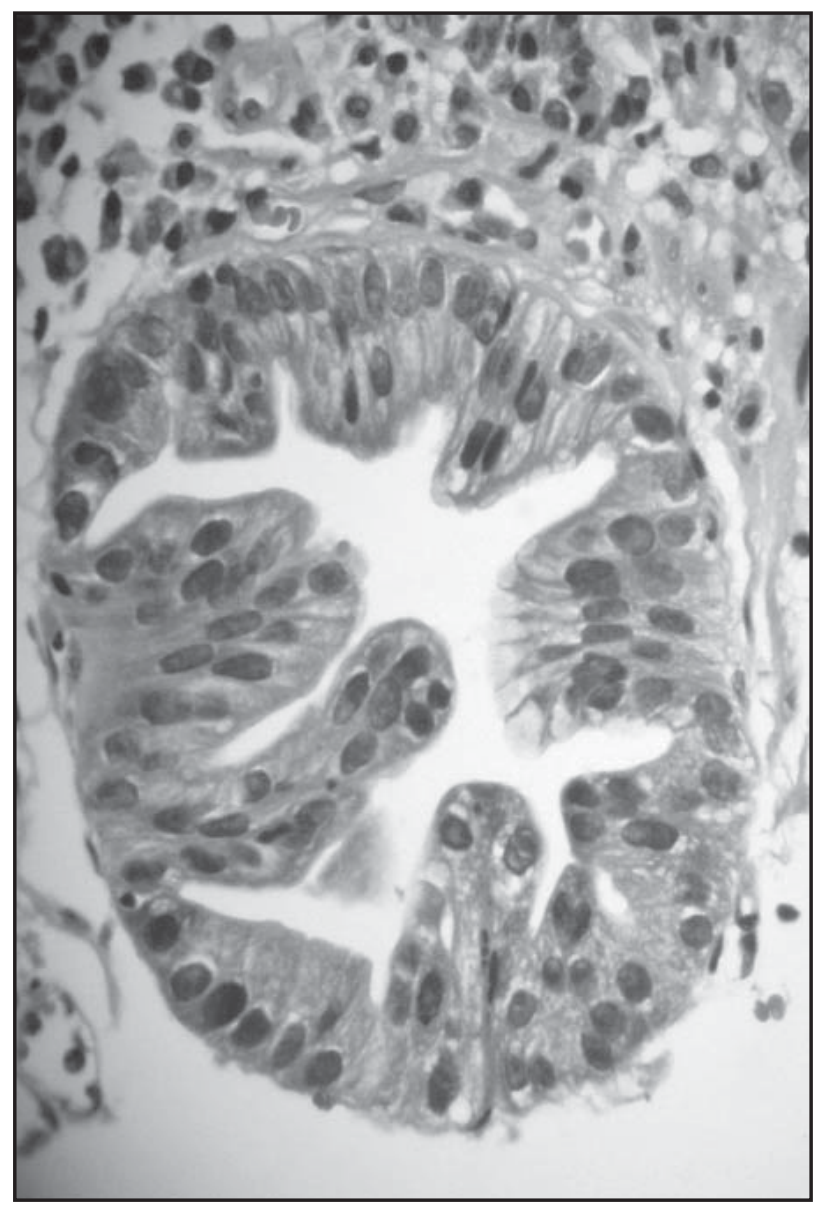

Figura 4 - Displasia de alto grau. Núcleos com discariose, hipercromasia e perda da polaridade (HE 400x)

\section{Esôfago de Barrett negativo para displasia}

No EB sem displasia, a arquitetura da mucosa e a maturação epitelial estão preservadas (Figura 1). A polaridade dos núcleos está mantida. Pode haver estratificação dos núcleos e mitoses nas glândulas profundas ou no colo foveolar. Os nucléolos, se presentes, devem ser pequenos. As células da superfície devem apresentar maturação quando comparadas com as células das porções profundas da $\operatorname{mucosa}{ }^{(9,11,19)}$. Pode haver perda da produção de mucinas nas células superficiais.

\section{Esôfago de Barrett indefinido para displasia}

O diagnóstico indefinido para displasia deve ser feito quando não se tem certeza de que as alterações morfológicas são displásicas ${ }^{(9,19)}$. As células das porções profundas da biópsia apresentam atipias (Figura 2) sugestivas de displasia, mas ocorre maturação na superfície da mucosa. Quando os cortes histológicos são tangenciais e não permitem estimar a maturação da base para a superfície da mucosa, deve-se optar pelo diagnóstico de indefinido para displasia. Outra possibilidade é a presença de atipias citológicas expressas como hipercromasia e pleomorfismo dos núcleos no contexto de inflamação e reparação da mucosa, ou mesmo na ausência de atividade inflamatória em alterações residuais após tratamento. Deve ser feito diagnóstico de indefinido para displasia e indicado acompanhamento endoscópico com biópsias para novas avaliações.

\section{Esôfago de Barrett com displasia de baixo grau}

No EB com displasia de baixo grau, a perda da maturação epitelial caracterizada pela presença de núcleos proporcionalmente maiores na superfície da mucosa é o principal atributo diagnóstico ${ }^{(9,11,19)}$. As células superficiais são similares às das glândulas subjacentes. Os núcleos exibem discariose e hipercromasia, mas permanecem ancorados à porção basal das células (Figura 3). Não há perda da polaridade dos núcleos. Pode haver estratificação dos núcleos similar à dos adenomas do cólon. Há redução do número de células caliciformes (Figura 3). A arquitetura da mucosa encontra-se em geral preservada. Pode haver distorção e aglomeramento de glândulas, mas o estroma entre as glândulas é evidente. A inflamação, se presente, deve ser mínima. O principal diagnóstico diferencial da displasia de baixo grau é feito em relação a reatividade e regeneração epitelial no contexto de inflamação da mucosa. Os casos com inflamação intensa e com atipias celulares sugestivas de displasia devem ser diagnosticados como indefinidos para displasia.

\section{Esôfago de Barrett com displasia de alto grau}

No EB com displasia de alto grau a perda da maturação epitelial é evidente, como na displasia de baixo grau. Há distorsão da arquitetura da mucosa com ramificação de criptas, podendo haver arranjos cribriformes e superfície viliforme $^{(9,19)}$. As atipias citológicas são mais acentuadas do que na displasia de baixo grau e se expressam com maior pleomorfismo e hipercromasia dos núcleos (Figura 4). Há mitoses, perda da polaridade e estratificação dos núcleos. As características histológicas da displasia de alto grau são similares às do carcinoma intramucoso(24). Como a variabilidade de interpretação constitui uma das principais limitações 
do diagnóstico patológico, recomenda-se que os casos de $E B$ com displasia sejam enviados à consulta para segunda opinião diagnóstica ${ }^{(1,24,27)}$. Há estudos que demonstram que o acordo entre os observadores é maior para as lesões com atipias citológicas de alto grau do que para as lesões de baixo grau ou negativas para displasia ${ }^{(17,18,24)}$.

\section{Problemas diagnósticos}

Um dos principais problemas é o diagnóstico excessivo de EB baseado na presença de epitélio glandular sem metaplasia intestinal(1,15). O contexto clínico é de um paciente com doença do refluxo gastroesofágico e hérnia de hiato. As biópsias demonstram epitélio glandular de corpo e fundo gástrico ou da cárdia, e o patologista faz diagnóstico de EB em mucosa do tipo juncional. Esse diagnóstico é incorreto. $O$ diagnóstico de $E B$ deve ser feito somente na presença de metaplasia intestinal ${ }^{(9,11,19)}$. Outro problema é o diagnóstico de EB em biópsias da cárdia apresentando metaplasia intestinal. O endoscopista não tem certeza do local da biópsia, se corresponde à EB curto ou à mucosa da cárdia que se deslocou cranialmente para o esôfago tubular. Neste caso, o diagnóstico deve ser descritivo. Deve ser investigada a possibilidade de comprometimento da cárdia em quadro de pangastrite associada a infecção por H. pylori(8).

Em síntese, o patologista desempenha papel fundamental para definir o diagnóstico de esôfago de Barrett e no acompanhamento dos pacientes para rastrear o desenvolvimento de displasia, que é o principal marcador morfológico de risco para câncer nesta entidade.

\section{Referências}

I. ALIKHAN, M. et al. Variable pathologic interpretation of columnar lined esophagus by general pathologists in community practice. Gastrointestinal Endoscopy, v. 50, p. 23-6, 1999.

2. ANDREOLLO, N. A. et al. Incidência e epidemiologia do epitélio de Barrett no Gastrocentro/Unicamp. Arq Gastroenterol, v. 34, p. 22-6, 1997.

3. ANTONIOLI, D. A.; WANG, H. H. Morphology of Barrett's esophagus and Barrett's-associated dysplasia and adenocarcinoma. Gastroenterol Clin N Am, v. 26, p. 495-506, 1997.

4. BORHAN-MANESH, F.; FARNUM, J. B. Incidence of heteropic gastric mucosa in the upper esophagus. Gut, v. 32, p. 96872, 1991.

5. CAMERON, A. J.; CARPENTER, H. A. Barrett's esophagus, highgrade dysplasia, and early adenocarcinoma: a pathological study. Am J Gastroenterol, v. 92, p. 586-91, 1997.

6. GEBOES, K.; VAN EYKEN, P. The diagnosis of dysplasia and malignancy in Barrett's esophagus. Histopathology, v. 37, p. 99-107, 2000.

7. GLICKMAN, J. N. et al. Phenotype of Barrett's esophagus and intestinal metaplasia of the distal esophagus and gastroesophageal junction: an immunohistochemical study of cytokeratins 7 and 20, Das- I and 45 Ml. Am J Surg Pathol, v. 25, n. I, p. 94-7, 200 I.

8. GOLDBLUM, J. R. et al. Inflammation and intestinal metaplasia of the gastric cardia: the role of gastroesophageal reflux and $H$. pylori infection. Gastroenterology, v. I 14, p. 633-9, 1998.

9. GOLDBLUM, J. R. Barrett's esophagus and Barrett's related dysplasia. Mod Pathol, v. 16, n. 4, p. 31 6-24, 2003.

I0. GOTTFRIED, M. R. et al. Incomplete intestinal metaplasia in the diagnosis of columnar lined esophagus (Barrett's esophagus). Am J Clin Pathol, v. 92, p. 74I-6, 1989.

II. HAGGITT, R. C. Barrett's esophagus, dysplasia and adenocarcinoma. Hum Pathol, v. 25, p. 982-93, 1994.
12. HAGGITT, R. C. Pathology of Barrett's esophagus.J Gastrointest Surg, v. 4, p. I 17-8, 2000.

13. JACOBS, E.; DEHOU, M. F. Heterotopic gastric mucosa in the upper esophagus: a prospective study of 33 cases and review of literature. Endoscopy, v. 29, n. 8, p. 71 10-5, 1997.

I4. JANKOWSKI, J. A. et al. Molecular evolution of the metaplasiadysplasia-adenocarcinoma sequence in the esophagus. Am J Pathol, v. I54, p. 965-73, 1999.

15. KIM, S. L. et al. Diagnosis inconsistencies in Barrett's esophagus. Gastroenterology, v. 107, p. 945-9, 1994.

16. LAPERTOSA, G. et al. Mucin histochemistry analysis in the interpretation of Barrett's esophagus: results of a multicenter study. Am J Clin Pathol, v. 98, p. 61-6, 1992.

17. MONTGOMERY, E. et al. Reproducibility of the diagnosis of dysplasia in Barrett's esophagus: a reafirmation. Hum Pathol, v. 32, p. 368-78, 200।

18. MONTGOMERY, E. et al. Dysplasia as a predictive marker for invasive carcinoma in Barrett's esophagus: a follow-up study based 138 cases from a diagnostic variability study. Hum Pathol, v. 32, p. 379-88, 200 I.

19. MONTGOMERY, E. Update on grading dysplasia in Barrett's esophagus. Pathology Case Reviews, v. 7, n. I, p. 35-42, 2002.

20. NANDUKAR, S. et al. Short segment Barrett's esophagus: prevalence, diagnosis and associations. Gut, v. 40, p. 710-5, 1997.

21. OFFNER, F. A. et al. Metaplastic columnar cells in Barrett's esophagus: a common and neglected cell type. Hum Pathol, v. 27, p. 885-9, 1996.

22. ORMSBY,A. H. et al. Cytokeratin subsets can reliably distinguish Barrett's esophagus from intestinal metaplasia of the stomach. Hum Pathol, v. 30, p. 288-94, 1999.

23. ORMSBY, A. H. et al. Cytokeratin immunoreactive patterns in the diagnosis of short-segment Barrett's esophagus. Gastroenterology, v. I19, p. 683-90, 2000. 
24. ORMSBY, A. H. et al. Observer variation in the diagnosis of superficial adenocarcinoma. Gut, v. 5I, p. 67I-6, 2002.

25. PAULL, A. et al.The histologic spectrum of Barrett's esophagus. N Engl J Med, v. 295, p. 476-80, 1976.

26. PETRAS, R. E. et al. Barrett's esophagus: a review of the pathologist's role in diagnosis and management. Pathol Annu, v. 26, p. I-32, 199|.

27. REID, B. J. et al. Observer variation in the diagnosis of dysplasia in Barrett's esophagus. Hum Pathol, v. 19, p. 166-78, 1988.
28. RUSCH, V. W. et al. The management of high-grade dysplasia and early cancer in Barrett's esophagus. Cancer, v. 74, p. $1225-9,1997$.

29. RIDDEL, R. H. et al. Dysplasia in inflammatory bowel disease: Standardized classification with provisional clinical implications. Human Pathol, v. 14, p. 931-68, 1983.

30. SPECHLER, S. J. Barrett's esophagus. N Engl J Med, v. 315 , p. 362-7I, 1986. 